\title{
1 DIRECTIONS IN INFORMATION SYSTEMS DEVELOPMENT: INTEGRATING NEW TECHNOLOGIES, RESEARCH APPROACHES, AND DEVELOPMENT PRACTICES
}

\author{
Nancy L. Russo \\ Department of Operations Management and Information Systems \\ Northern Illinois University \\ DeKalb, Illinois 60115 \\ U.S.A. \\ Brian Fitzgerald \\ Department of Accounting, Finance and Information Systems \\ National University of Ireland-Cork \\ Cork \\ Ireland
}

Information systems development (ISD) continues to be an issue of central significance and concern in the IS field-arguably the core topic for the field. Even as new research topics emerge in tandem with the latest developments in technology, the core issue of how to develop systems to leverage these new technologies and topics remains as problematic as ever. Looking at electronic commerce, one of the most recent areas to have stimulated much research interest, we can see that the work in this area is characterized initially by research that considers the basic functionality of these systems: what can they do? Then the focus moves to how to manage them: how can they be used to support organizational strategy? However, a fundamental principle-which may seem obvious, but is nevertheless not always stated-is that all of these systems have to be developed, and that someone must assume responsibility for doing that. Another fundamental principle is that identifying the purpose, functions, and structure of these systems is an extremely complex process.

Although some aspects of the development process have come to be more automated (supported by technology such as CASE tools, for example), the social and organizational issues remain as the crucially important ones. How do we get the right system for the organization? How does the process of development change? Who are the stakeholders? Even in the packaged

The original version of this chapter was revised: The copyright line was incorrect. This has been corrected. The Erratum to this chapter is available at DOI: 10.1007/978-0-387-35489-7_33 
software and component-based development areas, these issues are of major concern.

While the individual topics of the papers submitted to this Conference vary a great deal, in some way they all impinge on the area of information systems development. Whether the paper addresses new ways of viewing development, new methods, new models and frameworks, or examinations of political or social factors related to information systems, all of these things are related to how (and why) information systems are developed and implemented.

One of the stated goals of the conference was to help to bridge the gap between research and practice. Have we succeeded? We do see reflected in the Proceedings a large number of case studies, which reflects in-depth study of information systems development and/or use in practice. However, there are no papers that come purely from practice, which perhaps is not surprising considering the academic nature of the group.

In general, the papers included in the Conference address four primary questions: how do we develop, how do we manage, how do we understand, and how do we research information systems today? Of course, there is a great deal of overlap between these issues, but these broad thematic categories have been chosen to structure the presentation of the papers at the Conference and in these Proceedings.

\section{DEVELOPING INFORMATION SYSTEMS}

The papers in this section address the fundamental core of the field itselfinformation systems development-and question or challenge the most basic assumptions. A number of these papers call for new ways of thinking about information systems development, while others suggest new approaches to method creation and use. Common to all of the papers is an acknowledgment that previous approaches and methods are not satisfactory.

In "Accommodating Emergent Work Practices: Ethnographic Choice of Method Fragments," Baskerville and Stage question the assumption that information systems development methods and practice are closely aligned. They call for a recognition of the ISD process as being emergent, and provide a framework to enable the appropriate selection of method fragments in work practices which are emergent.

In "Information Systems Development as Flowing Wholeness," Cunha and Figueiredo argue from a similar vein, suggesting that information systems development should be understood as a continuous and holistic process. They identify a number of aspects, which characterize the current development environment, and argue that the necessity for completely new approaches to ISD is critical. 
Baskerville and Pries-Heje, in "Racing the E-Bomb: How the Internet Is Redefining Information Systems Development Methodology," also argue that the concept of ISD method needs to be altered. They identify a core set of ten properties for an ISD method geared to "Internet-time," and reinforce their argument with examples from three Danish companies.

Hedström and Eliason, in "The Use of Research-Based Information System Development Methods," also address the issue of method construction. They consider the use and adaptation of a specific method in practice, and propose a set of five "functions" (enlightenment, orientation, instrumental, confirmation, legitimization) which comprise a framework for investigating method use and adaptation.

In "Techniques and Methodologies for Multimedia Systems Development: A Survey of Industrial Practice," Lang and Barry focus on how multimedia systems are developed in practice. Using a survey, they confirm the finding that literature methods are not adopted in practice. They identify a trend toward increased incorporation of multimedia development in organizations, suggesting that research on multimedia development has been overshadowed in recent times by research on Web development.

Wynekoop, Johnson and Finan, in "Enterprise Network Design: How Is it Done?," consider the specific topic of enterprise network design. They point to the criticality of the enterprise network infrastructure and suggest that while development problems are further exacerbated in this area, "no systematic study" of this topic has been conducted, and they conclude with an agenda for future research in this area.

In "A Role-Based Framework for Information System Self-Development," Roost, Kuusik, and Veskioja describe a model of development in which IS development processes are performed in the context of organizational roles. A meta-model of the architecture of the IS development process and a general methodological framework for modeling and organizing such subject-centered development processes are presented.

\section{MANAGING INFORMATION SYSTEMS}

The papers in this section address the introduction, use, and management of information systems and information technologies. We see from these papers that new technologies challenge us to find new approaches to implementation, new business models, and new methods of evaluation.

In "Developing a Methodology to Evaluate the Impact of Staff Perceptions on the Strategic Value of Information Systems in a Small to Medium Sized Enterprise," Moreton and Aiken tackle the issue of information systems as a 
strategic resource for a SME. They hypothesize that employees have an incomplete understanding of organizational objectives, and propose a methodology to evaluate the effect that this incomplete perception has on the strategic impact of the organization.

Nandhakumar and Vidgen use actor network theory to examine the sociotechnical aspects of the introduction of new technology in "Due Process and the Introduction of New Technology: The Institution of Video Teleconferencing." They conclude that a more even-handed consideration of all actors-human and non-human - should be undertaken.

Allen and Kern examine the implementation of enterprise resource planning (ERP) systems in higher education institutions in "Enterprise Resource Planning Implementation: Stories of Power, Politics, and Resistance." Based on analysis from four case studies, they identify the following key issues: the organizational culture, past IT implementation characteristics, relationship and knowledge management, and existing power structures in organizations.

Four case sites are studied to provide understanding of web information system management, which includes enhancement, maintenance, and on-going development activity, in "Web Information Systems Management: Proactive or Reactive Emergence." In this paper, Eschenfelder and Sawyer use sociotechnical information networks to guide their analysis of the web IS management in the organizations studied.

In "Better Safe than Sorry? In Search of an Internet Business Model in Online Entertainment," Henfridsson, Holmström, and Hanseth describe the evolving strategies - both radical and conservative - undertaken by a Swedish computer game developer in the planning, development, and implementation of a new online game.

Brooks and Airey also consider online systems in "Consumer Privacy and Online Marketing: Bringing the Human Back into the Picture." They explore potential technical solutions that can allow marketers to obtain the information they need while acknowledging the privacy concerns of consumers. Technologies such as chat facilities and online telephone calls, for example, can provide a more human face to the virtual world.

The experiences and outcomes of applying a method for the development of an evaluation framework are described in "Observations from a Field Study on Developing a Framework for Pre-Usage Evaluation of CASE Tools," by Rehbinder, Lings, Lundell, Burman, and Nilsson. A framework was developed and successfully used at Volvo IT to evaluate a state-of-the-art CASE tool.

In "Software Process Maturity and Organizational Politics," Nielsen and Nørbjerg consider the issue of software process maturity, and identify deficiencies in the extant process maturity models. Their investigation involved action research in a particular company, which confirmed these deficiencies and 
that they hinder the achievement of an understanding of software development practice. They recommend a synthesis between the perspectives afforded by software process maturity and organizational politics.

In "Implications of a Service-Oriented View of Software," Layzell identifies a radical change in mindset of practitioners to view software as a service rather than a product. Such a shift has important implications for IS developers and managers. Layzell further argues that there is no method or set of tools that will achieve highly flexible, service-oriented software, a theme in keeping with several of the papers in the track on Developing Information Systems described earlier.

\section{RESEARCHING INFORMATION SYSTEMS}

The papers in this section provide new approaches to address research issues in information systems. By broadening the scope of concepts through which we conduct our research, we can increase our understanding of the field.

In "Method Diffusion as a Social Movement," Beynon-Davies and Williams focus on method diffusion, but abandon the normal rational perspective to address the phenomenon from a social, knowledge-based diffusion perspective. Drawing on a detailed analysis of the diffusion of the DSDM method, they propose six lessons that arise from adopting this research perspective.

Rose and Lewis combine structuration theory and action research to analyze an intranet development project in their paper "Using Structuration Theory in Action Research: An Intranet Development Project." This unique combination afforded richer insights into the ISD process and identified the necessity for changes to entrenched mental models that often arise in conventional ISD.

In "Cultivating Recalcitrance in Information Systems Research," Sørensen, Whitley, Madon, Klyachko, Hosein, and Johnstone conduct an initial theoretical exploration of the usefulness of applying the concept of recalcitrance in the context of empirical IS research. To do this, they consider how three research approaches can be adapted to allow for recalcitrance, and argue that adopting this perspective in the design and implementation of IS research can enrich and deepen empirical studies.

In his paper, "Implications of the Theory of Autopoiesis for the Discipline and Practice of Information Systems," Beeson argues for the importance of considering the discipline and practice of information systems from the perspective of autopoiesis theory. This, he suggests, would lead to a more radical reformulation of the discipline and its practice, principally in relation to altering the emphasis to a greater concern with co-operation and use issues. 
In "Absent Friends? The Gender Dimension in Information Systems Research," Adam, Howcroft, and Richardson argue that IS research fails to adequately address the concept of gender-a significant social factor affecting organization life-thus ignoring the existing body of gender and IT literature. The authors provide examples of this under-theorization of gender in both quantitative and qualitative studies.

Wilson, in her paper "A New Paradigm for Considering Gender in Information Systems Development Research," adopts an innovation perspective with a view to achieving a richer understanding of ISD; with the more specific aim of conceptualizing the gender dimension in IS research, which has received limited attention to date. The research is primarily literature driven, culminating in a stance against both technological and biological determinism.

In "Two Times Four Integrative Levels of Analysis: A Framework," Korpela, Mursu, and Soriyan draw on comparative psychology to propose a complex research framework with four levels (individual, group/activity, organizational, and societal) across two dimensions (intra-case and inter-case). Each of these levels and dimensions are discussed, and the implications for IS research and development are identified.

In "Thoughts on Studying Open Source Software Communities," Joe Feller addresses a current hot topic, open source software development, and discusses how it might be researched from an academic viewpoint. He considers the use of social scientific methods in the related fields of Information and Communication Technology (ICT), Computer-Mediated Communication (CMC), and Computer Supported Cooperative Work (CSCW). He poses as a critical question for future research the means by which OSS communities will survive and adapt to the growing commercial reality of OSS.

Sterner describes a knowledge management methodology framework in "Managing Knowledge Development in the Network Economy: Methodological Contributions." The framework brings together elements from evolutionary epistemology, the concept of tacit knowledge, and theories of systemic thinking and decision making.

\section{UNDERSTANDING INFORMATION SYSTEMS}

The papers in this section suggest new ways of looking at and thinking about information systems. They broaden our perspective, and help us see parallels and relationships between the public sector, including the realm of politics, and information systems development and implementation.

In their paper, "Defining Away the Digital Divide: A Content Analysis of Institutional Influences on Popular Representations of Technology," Kvasny and Truex conduct an analysis of political speeches delivered by U.S. government officials. In this analysis, they use Bourdieu's sociology of language to under- 
stand the popular representations of technology and its role in the new economy, and argue that symbolic violence is the dominant theme that emerges. In a broader sense, this should cause IS researchers to question our underlying assumptions about technology and consider the larger socio-political context in which our research takes place.

In "Doing Politics Around Electronic Commerce: Opposing the Regulation of Investigatory Powers Bill," Whitley and Hosein use Latour's model of political action to examine the resistance to legislation that was intended to support the development of electronic commerce in the UK. The process was analyzed in terms of the raising of new entities to be considered in the debate, determining which entities to take into consideration for action, and implementing the actions. The authors propose that these concepts can also be applied to the introduction of new information systems.

In their paper, "The Technology Imperative in Education," Bonner and Gopal warn of the danger of the technological imperative, echoing a theme in the Researching Information Systems stream. They stress the importance of preserving visibility in ISD and implementation to ensure this danger is avoided. The authors draw on the work of Beck and Latour to analyze a scenario in primary education in Canada, and suggest that such an analysis can provide a lens with which to ensure that similar outcomes do not occur in other organizational environments.

\section{CONCLUSIONS}

On an initial perusal of the papers presented in this Conference, one sees a very broad, possibly even disjointed set of topics. Upon closer inspection, however, a pattern begins to emerge. All of these papers are attempting to help us understand and address the current state of information technology in the world today.

The three panel sessions complement these papers. One panel asks us to question what we mean by "IT" in our research. Are we focusing too much on organizational actions and issues, and neglecting the information technologies embedded in the contexts we are studying? Another panel also addresses the area of research, and suggests that action research can help us build bridges between academia and practice. The third panel focuses on knowledge management and knowledge management systems to determine whether they are the latest "fad" or of real substance.

The overriding lesson to be learned from this gathering of ideas is that we as IS researchers (and practitioners) should questions assumptions, broaden our perspectives, and stretch our minds to meet the challenges of the new millennium. 


\section{About the Authors}

Nancy L. Russo is an associate professor of Information Systems at Northern Illinois University. She received her Ph.D. in Management Information Systems from Georgia State University in 1993. During 1998, she was a Visiting Professor in the Department of Accounting, Finance and Information Systems at University College Cork. In addition to on-going studies of the use and customization of system development methods in evolving contexts, her research has addressed IT innovation, research methods, and IS education issues. Her work has appeared in Information Systems Journal, Journal of Information Technology, Information, Technology \& People, System Development Management, Journal of Systems and Software, and various international conference proceedings. Nancy can be reached by e-mail at nrusso@niu.edu.

Brian Fitzgerald holds a statutory position in the Department of Accounting, Finance and Information Systems in University College Cork. He received his Ph.D. from the University of London and has held a position as Visiting Professor at Northern Illinois University. He is also Associate Editor for The Information Systems Journal, and DataBase, two leading international journals in the IS field. He has edited a number of books and has also published his work in several premier international journals and conferences. Prior to taking up an academic position, he spent more than ten years in industry working on information systems development. This experience was gained both in Ireland and abroad, and spans many business sectors. Brian can be reached by e-mail atbf@ucc.ie. 